\title{
Long noncoding RNA SPRY4-ITI promotes malignant development of colorectal cancer by targeting epithelial-mesenchymal transition
}

This article was published in the following Dove Press journal:

OncoTargets and Therapy

30 August 2016

Number of times this article has been viewed

\author{
Dong Cao' \\ Qiong Ding' \\ Wubin Yu' \\ Ming Gao' \\ Yilian Wang ${ }^{2}$ \\ 'Department of General Surgery, \\ The People's Hospital of Putuo, \\ Zhoushan, ${ }^{2}$ Department of \\ Cardiology, The Second People's \\ Hospital of Lianyungang, Xinpu, \\ People's Republic of China
}

Correspondence: Yilian Wang Department of Cardiology, The Second People's Hospital of Lianyungang, No 4I, Hailian East Road, Xinpu, Lianyungang, Jiangsu 222006,

People's Republic of China Email wangyilian664@I26.com

\begin{abstract}
The clinical significance and biological functions of long noncoding RNA SPRY4 intronic transcript 1 (SPRY4-IT1) in colorectal cancer (CRC) remain largely unclear. Herein, we are the first to report that the SPRY4-IT1 was significantly upregulated in CRC tissues, serum, and cells. Higher SPRY4-IT1 expression was markedly associated with advanced Tumor Node Metastasis (TNM) stage in a cohort of 84 CRC patients. Multivariate analyses indicated that SPRY4-IT1 expression could be useful as an independent predictor for overall survival. Further in vitro experiments revealed that knockdown of SPRY4-IT1 inhibited the proliferation, migration, and invasion of CRC cells and induced cell cycle arrestment. Moreover, we confirmed that the expression of epithelial-mesenchymal transition-related genes was modulated through alteration of SPRY4-IT1 expression. These results suggest that SPRY4-IT1, as an oncogenic regulator, may serve as a candidate prognostic marker and potential target for CRC therapies.
\end{abstract}

Keywords: long noncoding RNA, SPRY4-IT1, colorectal cancer, survival

\section{Introduction}

Colorectal cancer (CRC) is one of the most frequent malignancies and a common cause of cancer deaths worldwide. ${ }^{1} \mathrm{CRC}$ is becoming more prevalent in developing countries, especially in the People's Republic of China. ${ }^{2}$ Initiation of CRC, involving multiple genomic and epigenomic variations, is a complex biological process over an extended time period. ${ }^{3}$ Despite advanced developments achieved in detection methods and treatment for CRC in the past decade, the overall survival (OS) rate remains unsatisfactory. ${ }^{4}$ An elevating incidence and a poor outcome of CRC encourage us to further unravel the underlying molecular mechanisms of CRC progression. Therefore, the identification of novel prognostic markers and effective individualized therapeutic targets is an urgent requirement for the early detection and treatment of CRC.

It is well known that only $2 \%$ of the total genome encodes proteins, whereas $>90 \%$ of the genome can be transcribed as noncoding RNAs. ${ }^{5}$ Long noncoding RNAs (lncRNAs), usually $>200 \mathrm{nt}$ in length and located in nuclear or cytosolic fractions, also have no protein-coding capacity. ${ }^{6}$ Mounting evidence revealed that lncRNAs act as oncogene or tumor suppressors in a disease- or tissue-specific manner in carcinogenesis. ${ }^{7-10}$ The expression of IncRNA SPRY4 intronic transcript 1 (SPRY4-IT1), transcribed from an intron of the SPRY4 gene, has been reported to be dysregulated in various cancers. The SPRY4-IT1 was first observed to be overexpressed in melanoma and modulated cell proliferation, invasion, and cell apoptosis. ${ }^{11}$ Subsequent studies indicated that high expression of SPRY4-IT1 was significantly associated with worse outcomes of esophageal squamous 
cell carcinoma, clear cell renal cell carcinoma, gastric cancer, and prostate cancer. ${ }^{12-15}$ Nevertheless, the role of SPRY4-IT1 and its prognostic significance in $\mathrm{CRC}$ remain unclear.

In the present study, we seek to determine the clinical significance of SPRY4-IT1 and further explore the potential molecular mechanism of dysregulated SPRY4-IT1 expression in colorectal carcinogenesis. We found that upregulation of SPRY4-IT1 in CRC tissues and cell lines predicted worse outcome for CRC patients. Furthermore, SPRY4-IT1 knockdown could inhibit cell growth by blocking cell cycle progression. Additionally, we demonstrated that loss of SPRY4-IT1 expression in CRC cell lines altered the expression levels of epithelial-mesenchymal transition (EMT)associated genes.

\section{Patients and methods Clinical samples}

CRC tissues and adjacent normal tissues were obtained from 84 patients who underwent resection of CRC at The People's Hospital of Putuo and The Second People's Hospital of Lianyungang. The clinical characteristics of CRC patients are detailed in Table 1. No patient received chemotherapy or radiotherapy prior to surgery. The follow-up periods ranged from 3 months to 36 months, with a median of 26 months. Follow-up studies involved physical examination, laboratory analysis, and computed tomography if necessary. In addition, we also collected serum samples from $88 \mathrm{CRC}$ patients and 98 ageand sex-matched healthy controls. All of the tissues and serum samples were immediately frozen in liquid nitrogen and kept at $-80^{\circ} \mathrm{C}$ until RNA analysis. This study was approved by the Human Research Ethics Committee of The Second People's Hospital of Lianyungang and written informed consent was obtained from each patient. All samples were handled anonymously according to ethical and legal standards.

\section{Cell culture}

Three CRC cell lines (HT-29, HCT-116, and SW-480) and a normal colon epithelium cell line (FHC) were purchased from Shanghai Cell Collection, Chinese Academy of Sciences (Shanghai, China). The cells were maintained in Dulbecco's Modified Eagle's Medium or Roswell Park Memorial Institute 1640 (Thermo Fisher Scientific, Waltham, MA, USA) medium supplemented with $10 \%$ fetal bovine serum (10\% FBS) in a humidified atmosphere at $37^{\circ} \mathrm{C}$ with $5 \% \mathrm{CO}_{2}$.

\section{RNA extraction and quantitative real- time polymerase chain reaction}

Total RNA was extracted from tissues or serum samples by Trizol reagent (Thermo Fisher Scientific) and Trizol
Table I Correlation between SPRY4-ITI expression and clinical characteristics

\begin{tabular}{|c|c|c|c|}
\hline Characteristics & $\begin{array}{l}\text { Tumor low } \\
\text { expression } \\
(n=48) N(\%)\end{array}$ & $\begin{array}{l}\text { Tumor high } \\
\text { expression } \\
(n=36) N(\%)\end{array}$ & $\begin{array}{l}\text { Chi-squared test, } \\
P \text {-value }\end{array}$ \\
\hline Sex & & & 0.557 \\
\hline Male & $29(60.42)$ & $24(66.67)$ & \\
\hline Female & $19(39.58)$ & $12(33.33)$ & \\
\hline Age (years) & & & 0.488 \\
\hline$<68$ & $25(52.08)$ & $16(44.44)$ & \\
\hline$\geq 68$ & $23(47.92)$ & $20(55.56)$ & \\
\hline Tumor location & & & 0.191 \\
\hline Colon & 31 (64.58) & $28(77.78)$ & \\
\hline Rectum & $17(35.42)$ & $8(22.22)$ & \\
\hline TNM & & & 0.044 \\
\hline I-II & $28(58.33)$ & $13(36.1 \mathrm{I})$ & \\
\hline III-IV & $20(4 I .67)$ & $23(63.89)$ & \\
\hline $\mathrm{T}$ & & & 0.020 \\
\hline TI-T2 & $10(20.83)$ & I (2.78) & \\
\hline T3-T4 & $38(79.17)$ & $35(97.22)$ & \\
\hline $\mathrm{N}$ & & & 0.847 \\
\hline No & $29(60.42)$ & $21(58.33)$ & \\
\hline $\mathrm{NI}-\mathrm{N} 2$ & $19(39.58)$ & 15 (4I.67) & \\
\hline$M$ & & & 0.005 \\
\hline Mo & $4 \mathrm{I}(85.42)$ & $21(58.33)$ & \\
\hline MI & 7 (14.58) & 15 (4I.67) & \\
\hline Grade & & & 0.139 \\
\hline GI-G2 & $42(87.5)$ & $27(75.0)$ & \\
\hline G3 & $6(12.5)$ & $9(25.0)$ & \\
\hline CEA & & & 0.064 \\
\hline$<5 \mathrm{ng} / \mathrm{mL}$ & 15 (31.25) & 5 (13.89) & \\
\hline$\geq 5 \mathrm{ng} / \mathrm{mL}$ & $33(68.75)$ & $31(86.11)$ & \\
\hline CA199 & & & 0.136 \\
\hline$<37 \mathrm{U} / \mathrm{mL}$ & $4 \mathrm{I}(85.42)$ & $26(72.22)$ & \\
\hline$\geq 37 \mathrm{U} / \mathrm{mL}$ & 7 (14.58) & $10(27.78)$ & \\
\hline
\end{tabular}

Abbreviation: SPRY4-ITI, SPRY4 intronic transcript I.

LS reagent (Thermo Fisher Scientific) as described in our previous study. ${ }^{16} \mathrm{cDNA}$ was synthesized using the PrimeScript RT reagent Kit with gDNA Eraser (Takara, Dalian, People's Republic of China). Quantitative real-time polymerase chain reaction was performed using the SYBR Green-based polymerase chain reaction (Takara) using an ABI 7500 System (Thermo Fisher Scientific), as described previously. ${ }^{16}$ Glyceraldehyde 3-phosphate dehydrogenase was considered as an internal control. Fold changes were calculated by relative changes $\left(2^{-\Delta \Delta \mathrm{Ct}}\right)$. The sequence of SPRY4-IT1 primer was forward 5'-AGCCACATAAATTCAGCAGA-3', reverse 5'-CGATGTAGTAGGATTCCTTT-CA-3'.

\section{Transfection of small interfering RNA}

According to the expression of SPRY4-IT1 in CRC cell lines, we chose HCT-116 and SW-480 cells for the knockdown study. For knockdown of SPRY4IT1 expression, the nucleotide sequences of siRNA (SPRY4-IT1-1: CCCAGAATGTTGACAGCTGCCTCTT; SPRY4-IT1-2: GCTTTCTGATTCCAAGGCCTATTAA) were 
chemically synthesized and transfected into HCT-116/SW-480 cells using Lipofectamine 2000 (Thermo Fisher Scientific) for 48 hours. The negative control was transfected in parallel. The cells were then subjected to further functional assays.

\section{Cell proliferation assay}

After transfection for 48 hours, the cells were reseeded into 96-well plates $\left(5 \times 10^{3}\right.$ cells per well). Cell viability was measured using a cell counting kit 8 (CCK8; Dojindo, Rockville, MD, USA) according to the manufacturer's instructions. CCK8 solution was added to each well. The absorbance was evaluated at $450 \mathrm{~nm}$ and recorded on a Microplate Reader Victor (PerkinElmer Inc., Waltham, MA, USA).

\section{Cell cycle analysis}

The cells were fixed with $70 \%$ ethanol after transfection for 48 hours. Cells $\left(5 \times 10^{5}\right)$ were resuspended in $0.5 \mathrm{~mL}$ phosphatebuffered saline and supplemented with propidium iodide and $1 \mu \mathrm{g} / \mathrm{mL}$ RNase for 30 minutes. Treated samples were analyzed with a Beckman Coulter FC500 (Beckman Coulter; Brea, CA, USA). The experiments were performed in triplicate.

\section{Cell migration and invasion assays}

Cell migration and invasion were evaluated in cells incubated for 48 hours using non-Matrigel-coated or Matrigel-coated transwell cell culture chambers (BD Matrigel Invasion Chamber; BD Biosciences, San Jose, CA, USA) of $8 \mu \mathrm{m}$ pore size following the manufacturer's instruction. For migration assay, the cells were reseeded in the top chamber without Matrigel supplemented with $100 \mu \mathrm{L}$ serum-free medium, and $600 \mu \mathrm{L}$ of $10 \%$ FBS containing medium was added to the lower chamber. After 24 hours, cells were fixed with methanol and stained with crystal violet. Cells on the upper surface were removed using a cotton swap. For invasion assay, the cells were reseeded in the top chamber coated with Matrigel supplemented with $100 \mu \mathrm{L}$ serum-free medium, and $600 \mu \mathrm{L}$ of $20 \%$ FBS containing medium was added to the lower chamber as a chemoattractant. After incubation for 24 hours, cells that invaded the lower chamber were fixed and stained as described earlier. The number of migrating or invading cells was calculated under the microscope in five random fields and shown as the average per field.

\section{Western blot assay}

Cellular proteins were extracted with RIPA protein extraction reagent (Beyotime, People's Republic of China) and separated in $10 \%$ sodium dodecyl sulfate polyacrylamide gel and transferred onto a Polyvinylidene Fluoride membrane (EMD Millipore, Billerica, MA, USA), and were then blocked overnight with $5 \%$ nonfat dried milk containing antibodies against E-cadherin (BD Biosciences; 1:1,000 dilution), vimentin (Abcom, Danvers, USA; 1:1,000 dilution), or glyceraldehyde 3-phosphate dehydrogenase (Santa Cruz Biotechnology Inc., Dallas, TX, USA; 1:1,000 dilution) at $4^{\circ} \mathrm{C}$. The proteins were quantified by densitometry using Quantity One software (Bio-Rad, CA, USA).

\section{Statistical analysis}

Continuous data were expressed as mean \pm SD. Category data were presented as frequencies and percentages. Optimal cutoff values of SPRY4-IT1 were calculated by the receiver operating characteristic curve analysis. The $\chi^{2}$ test, Fisher's exact test, or Student's $t$-test were used for comparisons between groups if necessary. The OS was defined as the interval between the dates of surgery and death. OS rates were evaluated using the Kaplan-Meier method and log-rank test. The Cox proportional hazards model was used to further assess survival data. Age, sex, and the significant variables in univariate analysis were fitted into multivariate analysis of Cox regression analyses. Nomogram for OS was constructed by applying R 3.0.3 software (Institute for Statistics and Mathematics, Vienna, Austria). Its predictive efficiency was estimated by Harrell's concordance index (C-index). A calibration curve was established by plotting actual survival and predicted survival probability for patients stratified by predicted risk assessed by the prediction accuracy of the nomogram. $P<0.05$ was considered as statistical significance.

\section{Results \\ Expression of SPRY4-ITI is upregulated in CRC}

SPRY4-IT1 expression was measured in 84 paired CRC tissues and corresponding adjacent normal tissues by quantitative realtime polymerase chain reaction. We found that expression of SPRY4-IT1 was markedly overexpressed in CRC tissues compared with the corresponding normal tissues $(P<0.001$, Figure 1A). To further observe the expression pattern of SPRY4-IT1, we detected the levels of SPRY4-IT1 in serum samples of 88 $\mathrm{CRC}$ and 98 healthy controls. The results showed that expression levels of SPRY4-IT1 were also significantly upregulated in serum samples of CRC patients compared with healthy controls in accordance with CRC tissues (Figure 1B).

\section{Correlation between SPRY4-ITI expression and clinical characteristics in CRC}

To assess the correlation of SPRY4-IT1 expression with clinical data, according to the relative expression of SPRY4-IT1 
A

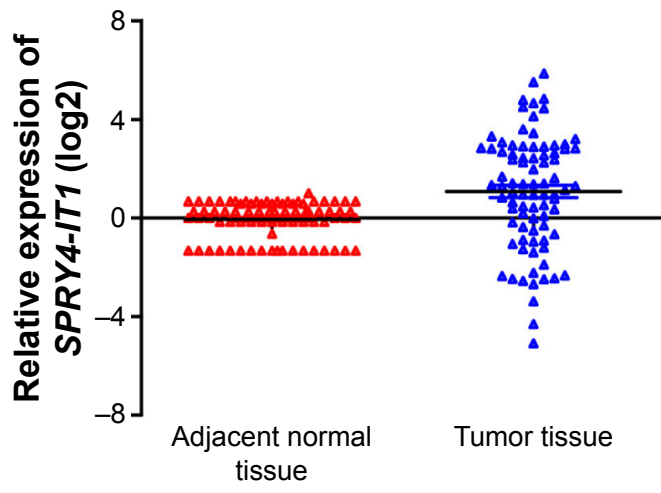

B

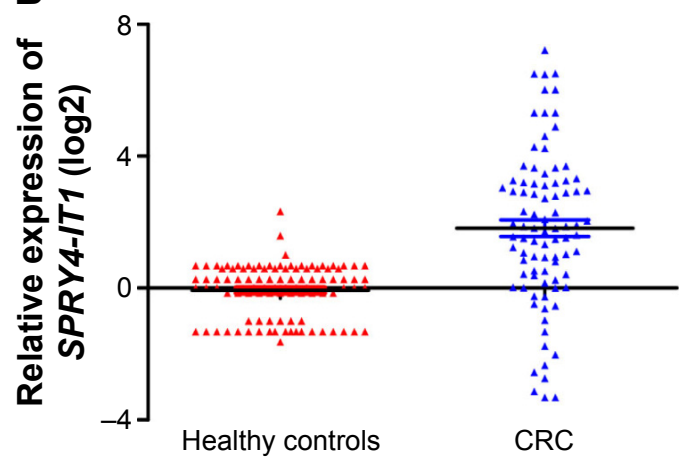

Figure I Relative expression of SPRY4-ITI in CRC tissues and serum samples.

Notes: (A) Relative SPRY4-ITI expression in 84 paired CRC tissues and corresponding adjacent normal tissues. (B) Relative SPRY4-ITI expression in serum samples of 88 CRC and 98 healthy controls. SPRY4-ITI expression was measured by qRT-PCR and normalized to GAPDH expression. The results are expressed as the fold change. Abbreviations: SPRY4-ITI, SPRY4 intronic transcript I; CRC, colorectal cancer; qRT-PCR, quantitative real-time polymerase chain reaction; GAPDH, glyceraldehyde 3-phosphate dehydrogenase.

in CRC tissues, the optimal cutoff value of SPRY4-IT1 expression was 2.87 -fold for OS with the largest sensitivity (67.6\%) and specificity (74.0; Figure 2A). CRC patients were then classified into two groups: the relative high group ( $n=36$, fold change $\geq 2.87$ ) and the relative low group ( $n=48$, fold change $<2.87$ ).

To further understand the clinical significance of SPRY4-IT1 in CRC, we identified the potential relationships between SPRY4-IT1 expression and clinical characteristics. Associations between SPRY4-IT1 expression and patients' clinical features are summarized in Table 1. Noticeably, high expression of SPRY4-IT1 had a significant correlation with the advanced Tumor Node Metastasis (TNM) stage, depth of invasion, and metastasis.

\section{Overexpression of SPRY4-ITI is associated with poor prognosis of CRC patients}

To evaluate the prognostic value of SPRY4-IT1 in CRC patients, OS curve was plotted by the Kaplan-Meier analysis and log-rank test. We observed that patients with low expression of SPRY4-IT1 had a worse OS (Figure 2B). The 3-year survival rates were $77.1 \%$ and $36.1 \%$ for patients with low expression and high expression, respectively, and patients with high expression presented a shorter survival time (median: 18 months) compared with those with low expression (median: 31 months). Additionally, advanced TNM stage and distant metastasis were significantly correlated with worse OS (Figure $2 \mathrm{C}$ and D). These results suggested that high SPRY4-IT1 expression may represent a novel indicator of poor prognosis in CRC.

To further determine whether SPRY4-IT1 expression was a prognostic predictor for CRC patients, Cox regression analysis was performed. The results of univariate analyses are shown in Table 2. Further analysis in a multivariate Cox regression model indicated that SPRY4-IT1 expression together with distant metastasis was strongly associated with OS ( $P=0.002, P=0.001$, respectively). The results revealed that SPRY4-IT1 expression was an independent prognostic indicator for OS (Hazard ratio [HR] $=3.21,95 \%$ Confidence interval $[\mathrm{CI}]=1.55-6.67$ ) in CRC patients (Table 2).

To predict the clinical outcome of CRC patients after surgery, a predictive model was constructed by significant variables in multivariate analysis (Figure 3A). The nomogram could predict the probability of 3-year survival for CRC patients after surgery (C-index: 0.73 ). Additionally, an internal calibration was performed, and the calibration curve presents a good predictive match with the actual outcome (Figure 3B).

\section{Modulation of SPRY4-ITI expression in CRC cells}

To assess the biological roles of SPRY4-IT1 in CRC, we examined SPRY4-ITI expression in various cell lines. The results showed that SPRY4-IT1 expression markedly upregulated in the CRC cell lines (Figure 4A), especially SW-480 and HCT-116. To downregulate SPRY4-IT1 levels in CRC cells, small interference (si)-SPRY4-IT1 was transfected into SW-480 and HCT-116 cells. We found that SPRY4-ITI expression was effectively blocked in both si-SPRY4-ITItransfected SW-480 and HCT-116 cells compared with sinegative control (NC) cells.

\section{SPRY4-ITI promotes CRC cell growth by accelerating the cell cycle progression}

The significant upregulation in SPRY4-IT1 expression encouraged us to explore the possible biological effect of 

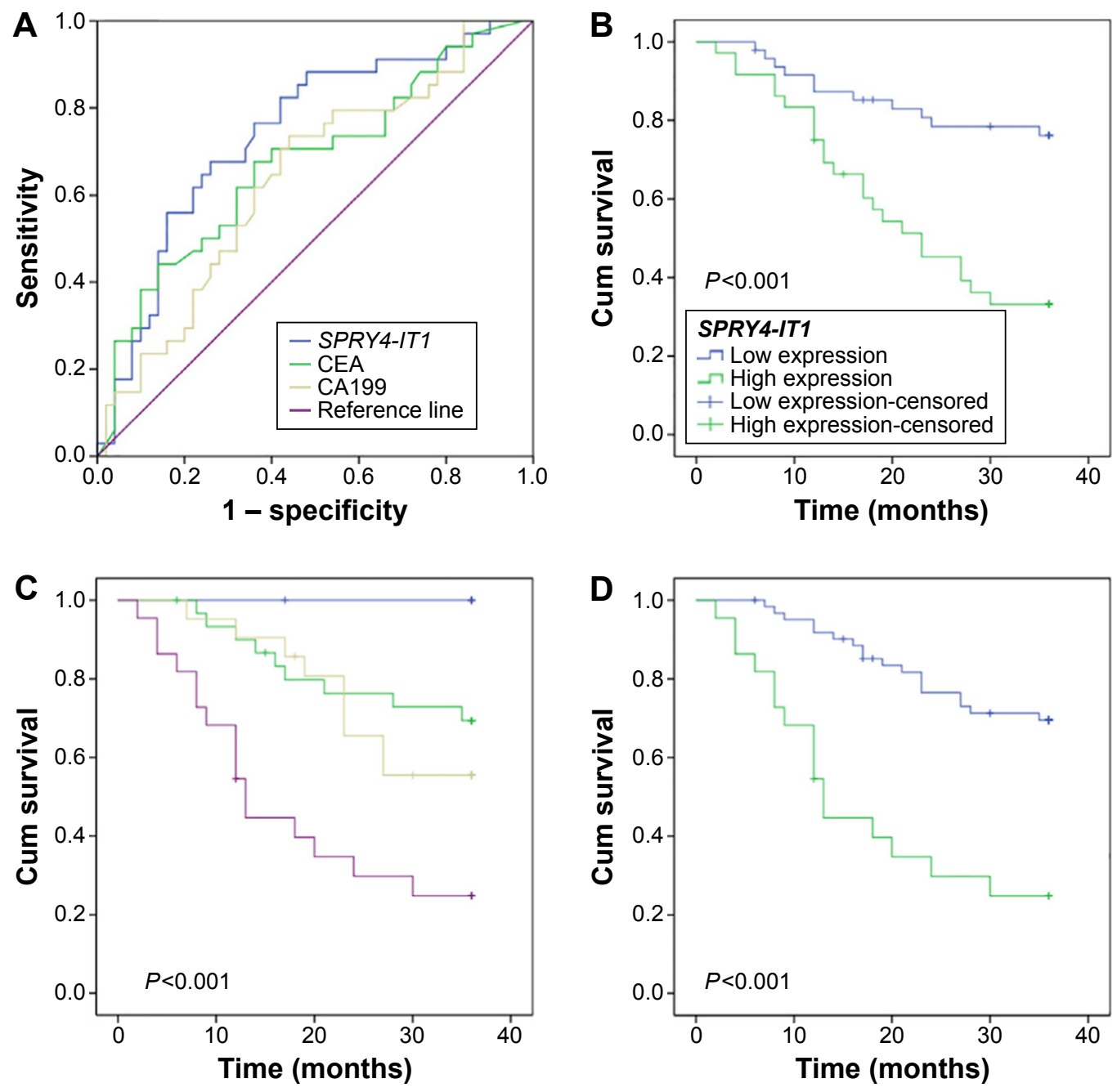

\begin{tabular}{|ll|}
\hline TNM stage & \\
$\neg$ I & + I-censored \\
- II & + II-censored \\
- III & + III-censored \\
$\neg$ IV & + IV-censored \\
\hline
\end{tabular}

\begin{tabular}{|l|}
\hline Distant metastasis \\
$\neg$ Negative \\
$\neg$ Positive \\
+ Negative-censored \\
+ Positive-censored \\
\hline
\end{tabular}

Figure 2 ROC curve analysis and prognostic value of SPRY4-ITI in CRC patients.

Notes: (A) ROC analysis shows the predictive capability. Kaplan-Meier analysis of overall survival was analyzed according to the SPRY4-ITI expression levels (B), TNM stage (C), and distant metastasis (D).

Abbreviations: ROC, receiver operating characteristic curve; SPRY4-ITI, SPRY4 intronic transcript I; CRC, colorectal cancer; Cum, cumulative; TNM, Tumor Node Metastasis.

Table 2 Summary of overall survival analyses by univariate and multivariate Cox regression analysis

\begin{tabular}{|c|c|c|c|c|c|}
\hline \multirow[t]{2}{*}{ Risk factors } & \multirow[t]{2}{*}{ Category } & \multicolumn{2}{|c|}{ Univariate analysis } & \multicolumn{2}{|c|}{ Multivariate analysis } \\
\hline & & HR (95\% Cl) & $P$-value & HR (95\% Cl) & $P$-value \\
\hline Sex & $F(n=3 \mid) / M(n=53)$ & $1.09(0.54-2.20)$ & 0.816 & $1.50(0.72-3.13)$ & 0.274 \\
\hline Age (years) & $\geq 68(n=43) /<68(n=4 I)$ & $0.97(0.50-1.90)$ & 0.931 & $0.74(0.37-1.49)$ & 0.399 \\
\hline Tumor location & Colon $(n=59) /$ rectum $(n=25)$ & $1.25(0.60-2.62)$ & 0.553 & & \\
\hline $\mathrm{T}$ & T3-T4 $(n=73) / T I-T 2(n=I I)$ & $6.32(0.86-46.22)$ & 0.069 & & \\
\hline $\mathrm{N}$ & NI-N2 (n=34)/N0 (n=50) & $1.79(0.91-3.50)$ & 0.092 & & \\
\hline M & $M I(n=22) / M 0(n=62)$ & $4.31(2.18-8.52)$ & $<0.001$ & $3.44(1.7 \mid-6.92)$ & 0.001 \\
\hline Grade & G3 $(n=15) / G 1-G 2(n=69)$ & $1.86(0.84-4.12)$ & 0.125 & & \\
\hline CEA & $\geq 5(n=20) /<5(n=64)$ & $1.61(0.66-3.88)$ & 0.293 & & \\
\hline CA199 & $\geq 37(n=17) /<37(n=67)$ & $1.45(0.68-3.11)$ & 0.338 & & \\
\hline SPRY4-ITI & High $(n=36) /$ low $(n=48)$ & $3.72(1.80-7.67)$ & $<0.001$ & $3.21(1.55-6.67)$ & 0.002 \\
\hline
\end{tabular}

Notes: Results are in response to Cox regression analysis $(P<0.05)$.

Abbreviations: SPRY4-ITI, SPRY4 intronic transcript I; HR, hazard ratio; Cl, confidence interval; T, depth of invasion; N, lymph node; M, distant metastasis. 
A

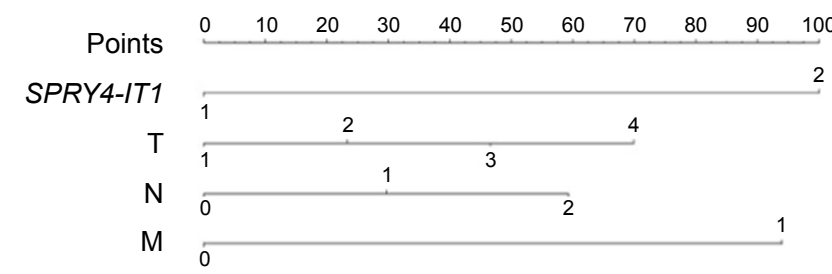

Total points $\begin{array}{llllllllllllll}20 & 40 & 60 & 80 & 100 & 120 & 140 & 160 & 180 & 200 & 220 & 240 & 260\end{array}$

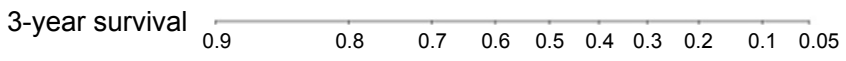

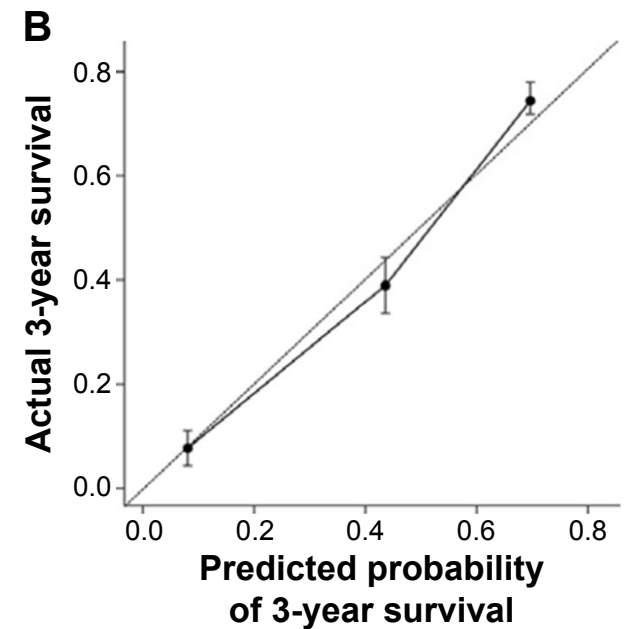

Figure 3 A survival predictive model in CRC.

Notes: (A) Nomogram for survival of CRC patients prior to surgery. (B) Calibration curve for 3-year survival. The solid line represents performance of the actual nomogram, and the dashed line shows an ideal nomogram. Error bars indicate $95 \% \mathrm{Cl}$.

Abbreviations: CRC, colorectal cancer; SPRY4-ITI, SPRY4 intronic transcript I; T, depth of invasion; N, lymph node; M, distant metastasis.

A

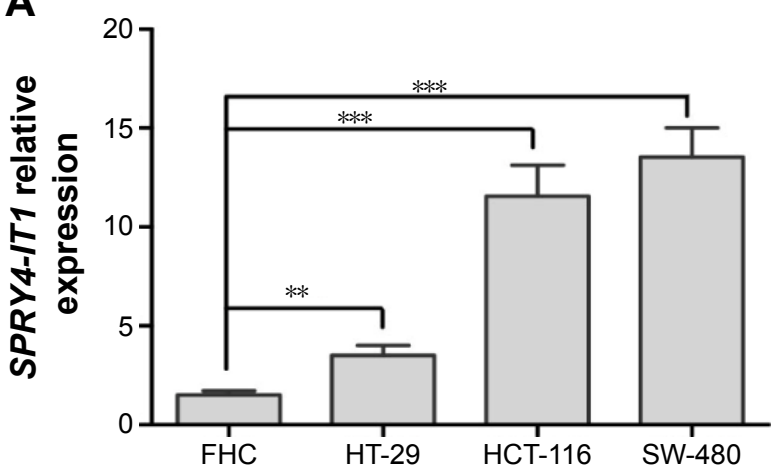

C

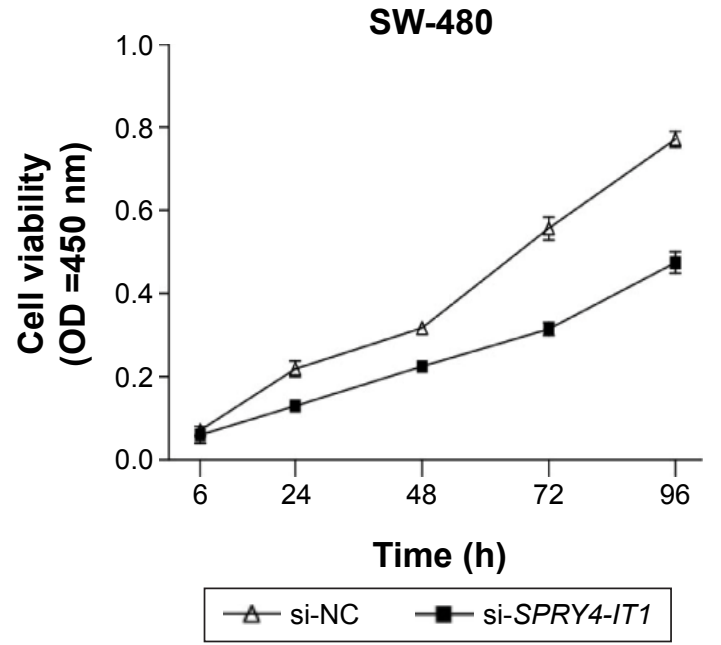

B

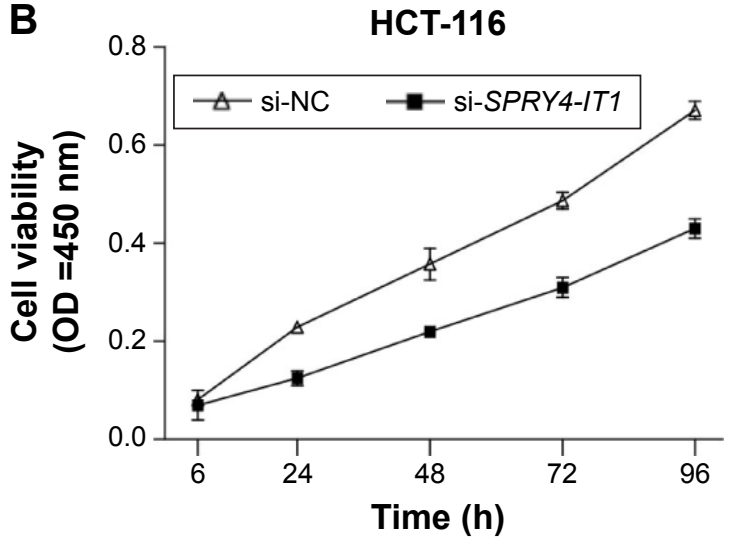

D

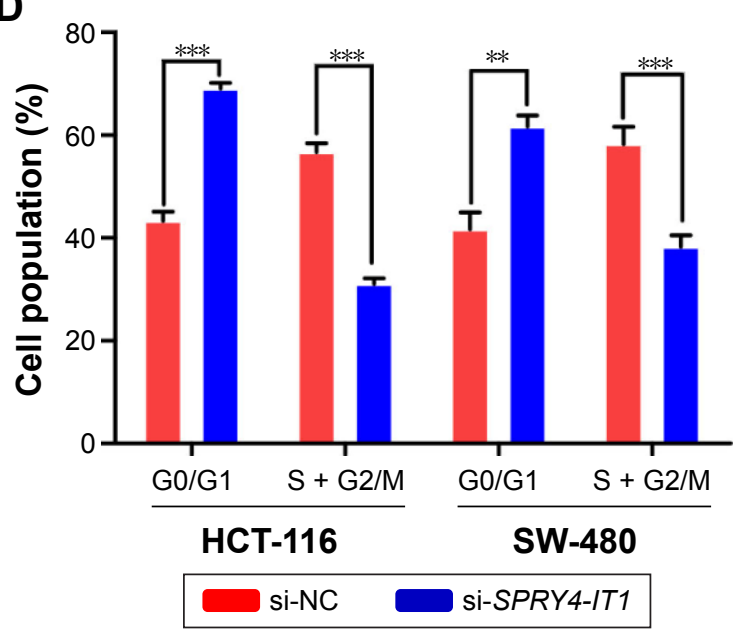

Figure 4 Effect of SPRY4-ITI on CRC cell proliferation.

Notes: (A) Expression of SPRY4-ITI in CRC cell lines and a normal human colon epithelial cell line (FHC). Cell viability of HCT-II6 (B) and SW-480 (C) when SPRY4-ITI was silenced. (D) Cell cycle analysis of CRC cells transfected with si-NC or si-SPRY4-ITI. The data are presented as the mean \pm SD. The experiments are performed in triplicate. $* * P<0.01$, $* * * P<0.001$.

Abbreviations: si-SPRY4-ITI, small inference-SPRY4 intronic transcript I; CRC, colorectal cancer; OD, optical density; h, hours; si-NC, small interference-negative control. 

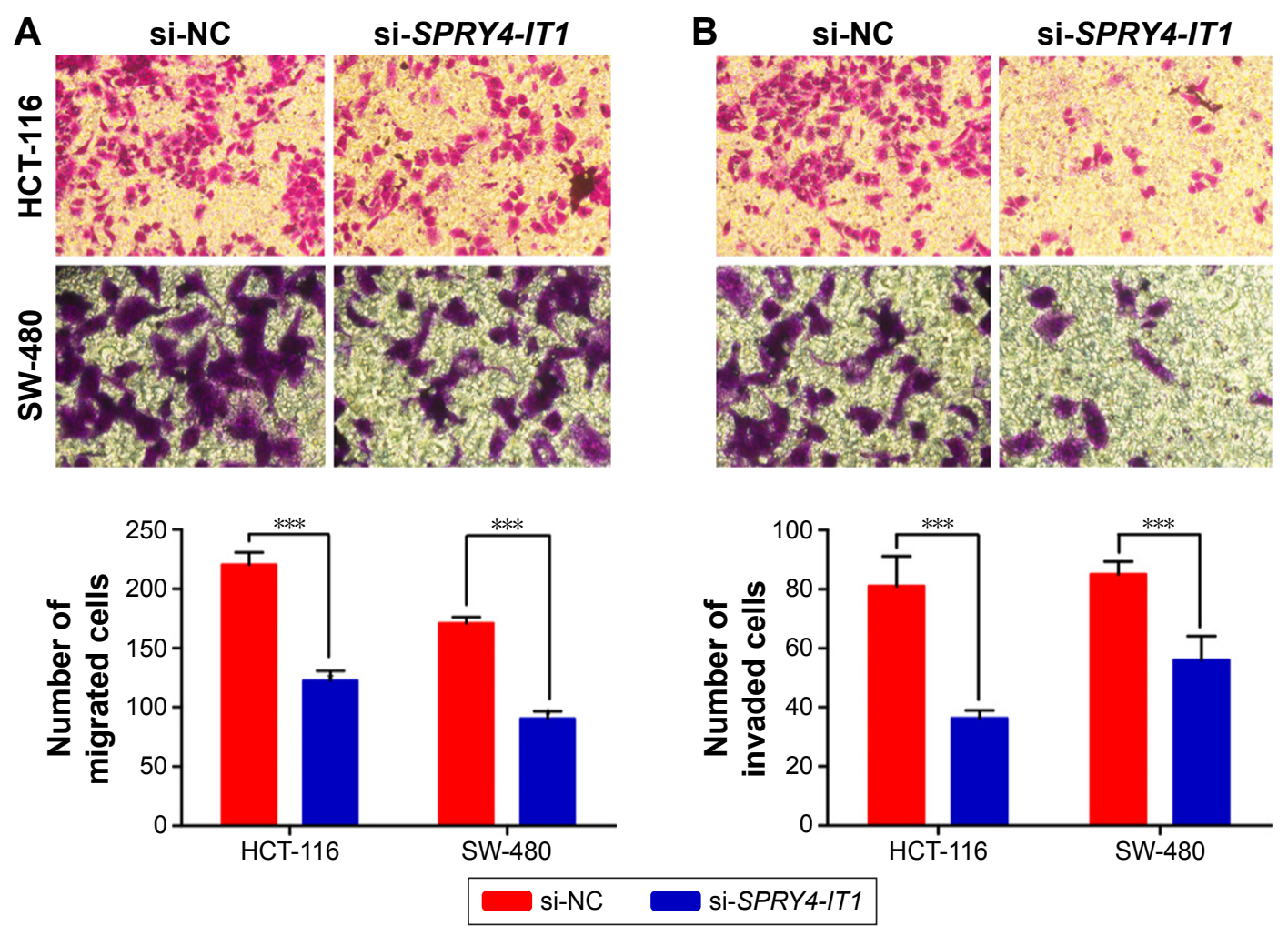

Figure 5 Effect of SPRY4-ITI on CRC cells migration and invasion.

Notes: Transwell assays were used to explore the changes in migratory $(\mathbf{A})$ and invasive $(\mathbf{B})$ abilities of CRC cells treated with si-NC or si-SPRY4-ITI. Results are in response to a Student's $t$ test. $* * * P<0.001$.

Abbreviations: si-SPRY4-ITI, small inference-SPRY4 intronic transcript I; CRC, colorectal cancer; si-NC, small interference-negative control.

SPRY4-IT1 in carcinogenesis. To investigate the biological function of SPRY4-IT1 in CRC, we first measured the effect of SPRY4-IT1 knockdown on cell proliferation by CCK8 assay. The results showed that the cell proliferation of HCT116 or SW-480 was significantly decreased in si-SPRY4-IT1 transfected cells compared with the corresponding controls (Figure 4B and C).

To further determine whether the effect of SPRY4-IT1 on cell proliferation of CRC is regulated by altering cell cycle progression, flow cytometry was carried out. The results showed that SPRY4-IT1 knockdown promoted a significant accumulation of cells at $\mathrm{G} 0 / \mathrm{G} 1$ phase and a significant decrease in cells in $\mathrm{S}+\mathrm{G} 2 / \mathrm{M}$ phase (Figure 4D). Taken together, SPRY4-IT1 promoted cell proliferation of CRC cells through modulation of the cell cycle progression.

\section{SPRY4-ITI promotes CRC cells migration and invasion}

The effect of SPRY4-IT1 knockdown on CRC cells migration or invasion was examined by transwell assays. Our results showed that SPRY4-IT1 knockdown suppressed cell migration by $44.5 \%$ in HCT- 116 cells and by $47.2 \%$ in
SW-480, respectively $(P<0.001$, Figure 5A). Furthermore, SPRY4-IT1 knockdown in CRC cells led to a significant decrease in cell invasion $(P<0.001$, Figure 5B). These data suggested that SPRY4-IT1 facilitated cell migration and invasion in vitro.

\section{SPRY4-ITI induces epithelial-mesenchymal transition in CRC cells}

As the EMT process was involved in cancer progression, our study also investigated the effect of SPRY4-IT1 on expression of the EMT-induced markers in CRC cells by Western blot. The results showed that SPRY4-IT1 knockdown inhibited E-cadherin expression and increased vimentin expression (Figure 6). Thus, SPRY4-IT1 promotes CRC cells metastasis partially through affecting the EMT process.

\section{Discussion}

In the present study, we first observed that SPRY4-IT1 expression was obviously upregulated in CRC tissues and serum samples compared with the corresponding normal tissues and healthy controls, respectively. Our results demonstrated that high expression of SPRY4-IT1 was associated with advanced 
A
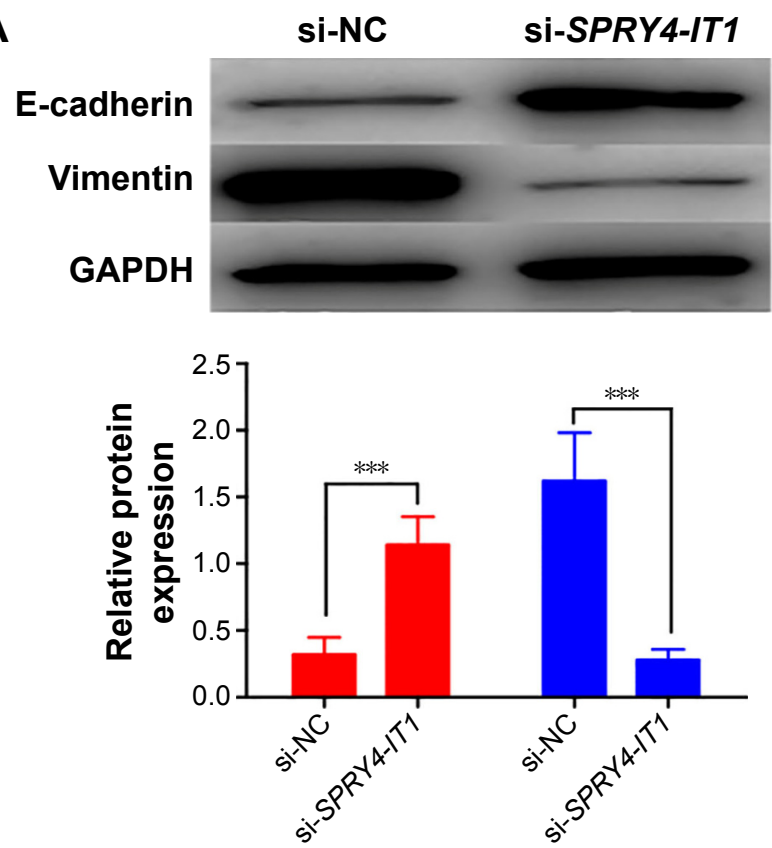

B
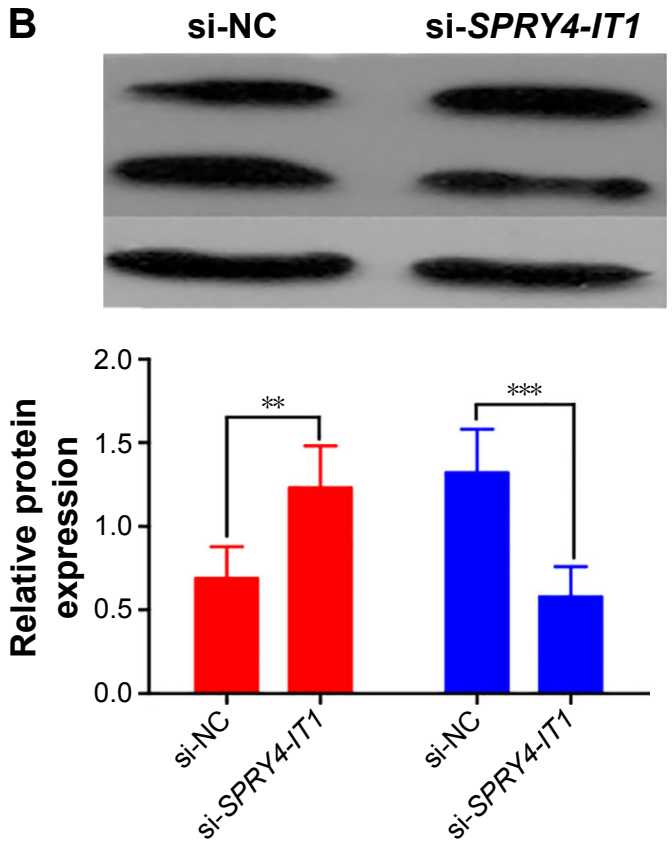

E-cadherin

Vimentin

Figure 6 Effect of SPRY4-ITI on CRC cells epithelial-mesenchymal transition.

Notes: (A) Western blot and densitometry analysis of E-cadherin and vimentin protein levels in HCT-II6 cells when SPRY4-ITI was inhibited. (B) Western blot and densitometry analysis of E-cadherin and vimentin protein levels in SW-480 cells when SPRY4-ITI was inhibited. Results are in response to a Student's $t$ test. $* * P<0.0 I$, $* * * P<0.001$.

Abbreviations: si-SPRY4-ITI, small inference-SPRY4 intronic transcript I; CRC, colorectal cancer; GAPDH, glyceraldehyde 3-phosphate dehydrogenase; si-NC, small interference-negative control.

TNM stage and distant metastasis. Furthermore, OS time of patients with high SPRY4-IT1 expression was significantly shorter than that of patients with low SPRY4-IT1 expression. Multivariate analyses further revealed that SPRY4-IT1 expression was an independent prognostic indicator in CRC patients. Additionally, SPRY4-IT1 knockdown inhibited the proliferation of CRC cells by blocking cell cycle progression. Moreover, SPRY4-IT1 knockdown suppressed the migration and invasion of CRC cells via affecting the EMT process.

IncRNAs are transcribed from the "desert region" of the genome as novel biomarkers monitoring disease recurrence or progression. ${ }^{17}$ Although some lncRNAs present a similar expression pattern and biological behavior, it is still necessary for us to understand different effects of lncRNAs underlying exact mechanisms on distinct cancer types. SPRY4-IT1 is reported to be a negative prognostic factor in various types of cancer, and it is overexpressed and acts as an oncogene in esophageal squamous cell carcinoma (ESCC), ${ }^{18}$ prostate cancer, ${ }^{15}$ and non small-cell lung cancer (NSCLC). ${ }^{19}$ However, it also functions as a tumor suppressor in gastric cancer. ${ }^{20}$ This difference could be due to a tissue-specific expression pattern of SPRY4-IT1 in different cancers. In our study, the expression of SPRY4-IT1, acting as an oncogene, increased in CRC tissues and serum samples. Furthermore, SPRY4-IT1 expression was identified to be an adverse prognostic indicator in CRC.

Previous studies have demonstrated that elevated SPRY4-IT1 expression leads to the abnormal expression of SPRY4-IT1 in different types of cancer cells, and the expression levels of SPRY4-IT1 were regulated by the polycomb group protein enhancer of zeste homolog $2^{21}$ and DNMT1. ${ }^{20}$ EMT is a key step toward cancer progression including CRC, and abnormal expression of E-cadherin and vimentin is a hallmark of the EMT process enhancing tumor cell motility. ${ }^{22}$ However, the underlying functions and mechanisms of SPRY4-IT1 in CRC remain unknown. In this study, we investigated the expression of SPRY4-IT1 in a panel of cell lines and performed a series of experiments to explore the functions of SPRY4-IT1 in CRC progression. We revealed that knockdown of SPRY4-IT1 impaired cell growth, invasion, and EMT process. Modulation of SPRY4IT1 expression can impact the protein levels of E-cadherin and vimentin, suggesting that SPRY4-IT1 affected CRC progression partly through the EMT process. The present study advances our understanding of the functions of SPRY4-IT1 as important regulators of CRC progression.

Emerging evidence showed that the prognostic model helps the physicians to identify high-risk patients to improve 
therapeutic strategies. Nomogram is reported to predict clinical outcome in patients with various types of cancers..$^{23,24}$ TNM and Duke staging systems are used to assess clinical outcome in CRC patients based on risks for their disease progression and death. We are also attempting to establish a predictive model to calculate the probability of 3-year OS for CRC patients according to SPRY4-IT1 expression and the significant variables in multivariate analyses. Our model performed well in predicting the clinical prognosis of CRC patients based on the $\mathrm{C}$-index $(0.73)$ and the calibration curve (Figure 3B). This model aims to calculate some of the heterogeneity within the TNM or Duke stages and provides an appropriate strategy for CRC management.

Several limitations should be acknowledged. First, the findings from this study will have to be validated in a large sample size. Second, longer follow-up periods are necessary to precisely evaluate OS of CRC patients. Finally, the effect of SPRY4-IT1 overexpression on CRC cells was not explored.

\section{Conclusion}

The present work provides the first link between SPRY4-IT1 expression and CRC progression. We proved that SPRY4-ITI was upregulated in CRC and its elevated expression may promote CRC carcinogenesis and development. These results revealed that SPRY4-IT1 may be a candidate prognostic marker and a target for CRC treatment.

\section{Disclosure}

The authors report no conflicts of interest in this work.

\section{References}

1. Jemal A, Bray F, Center MM, Ferlay J, Ward E, Forman D. Global cancer statistics. CA Cancer J Clin. 2011;61(2):69-90.

2. Chen W, Zheng R, Baade PD, et al. Cancer statistics in China, 2015. CA Cancer J Clin. 2016;66(2):115-132.

3. Walsh JM, Terdiman JP. Colorectal cancer screening: scientific review. JAMA. 2003;289(10):1288-1296.

4. Edwards BK, Noone AM, Mariotto AB, et al. Annual Report to the Nation on the status of cancer, 1975-2010, featuring prevalence of comorbidity and impact on survival among persons with lung, colorectal, breast, or prostate cancer. Cancer. 2014;120(9):1290-1314.

5. Chen LL, Carmichael GG. Long noncoding RNAs in mammalian cells: what, where, and why? Wiley Interdiscip Rev RNA. 2010;1(1):2-21.

OncoTargets and Therapy

\section{Publish your work in this journal}

OncoTargets and Therapy is an international, peer-reviewed, open access journal focusing on the pathological basis of all cancers, potential targets for therapy and treatment protocols employed to improve the management of cancer patients. The journal also focuses on the impact of management programs and new therapeutic agents and protocols on
6. Ponting CP, Oliver PL, Reik W. Evolution and functions of long noncoding RNAs. Cell. 2009;136(4):629-641.

7. Gutschner T, Diederichs S. The hallmarks of cancer: a long non-coding RNA point of view. RNA Biol. 2012;9(6):703-719.

8. Deng Q, He B, Gao T, et al. Up-regulation of $91 \mathrm{H}$ promotes tumor metastasis and predicts poor prognosis for patients with colorectal cancer. PLoS One. 2014;9(7):e103022.

9. Deng Q, Sun H, He B, et al. Prognostic value of long non-coding RNA HOTAIR in various cancers. PLoS One. 2014;9(10):e110059.

10. Han D, Gao X, Wang M, et al. Long noncoding RNA H19 indicates a poor prognosis of colorectal cancer and promotes tumor growth by recruiting and binding to eIF4A3. Oncotarget. Epub 2016 Mar 14.

11. Khaitan D, Dinger ME, Mazar J, et al. The melanoma-upregulated long noncoding RNA SPRY4-IT1 modulates apoptosis and invasion. Cancer Res. 2011;71(11):3852-3862.

12. Xie HW, Wu QQ, Zhu B, et al. Long noncoding RNA SPRY4-IT1 is upregulated in esophageal squamous cell carcinoma and associated with poor prognosis. Tumour Biol. 2014;35(8):7743-7754.

13. Zhang HM, Yang FQ, Yan Y, Che JP, Zheng JH. High expression of long non-coding RNA SPRY4-IT1 predicts poor prognosis of clear cell renal cell carcinoma. Int J Clin Exp Pathol. 2014;7(9):5801-5809.

14. Peng W, Wu G, Fan H, Wu J, Feng J. Long noncoding RNA SPRY4-IT1 predicts poor patient prognosis and promotes tumorigenesis in gastric cancer. Tumour Biol. 2015;36(9):6751-6758.

15. Mouraviev V, Lee B, Patel V, et al. Clinical prospects of long noncoding RNAs as novel biomarkers and therapeutic targets in prostate cancer. Prostate Cancer Prostatic Dis. 2016;19(1):14-20.

16. Ma B, Li M, Zhang L, et al. Upregulation of long non-coding RNA TUG1 correlates with poor prognosis and disease status in osteosarcoma. Tumour Biol. 2016;37(4):4445-4455.

17. Vidal AF, Sandoval GT, Magalhaes L, Santos SE, Ribeiro-Dos-Santos A. Circular RNAs as a new field in gene regulation and their implications in translational research. Epigenomics. 2016;8(4):551-562.

18. Xu Y, Qiu M, Chen Y, et al. Long noncoding RNA, tissue differentiationinducing nonprotein coding RNA is upregulated and promotes development of esophageal squamous cell carcinoma. Dis Esophagus. Epub 2016 Feb 2.

19. Hu X, Bao J, Wang Z, et al. The plasma lncRNA acting as fingerprint in non-small-cell lung cancer. Tumour Biol. 2016;37(3):3497-3504.

20. Xie M, Nie FQ, Sun M, et al. Decreased long noncoding RNA SPRY4IT1 contributing to gastric cancer cell metastasis partly via affecting epithelial-mesenchymal transition. J Transl Med. 2015;13:250.

21. Sun M, Liu XH, Lu KH, et al. EZH2-mediated epigenetic suppression of long noncoding RNA SPRY4-IT1 promotes NSCLC cell proliferation and metastasis by affecting the epithelial-mesenchymal transition. Cell Death Dis. 2014;5:e1298.

22. Thiery JP, Acloque H, Huang RY, Nieto MA. Epithelial-mesenchymal transitions in development and disease. Cell. 2009;139(5):871-890.

23. Callegaro D, Miceli R, Bonvalot S, et al. Development and external validation of two nomograms to predict overall survival and occurrence of distant metastases in adults after surgical resection of localised soft-tissue sarcomas of the extremities: a retrospective analysis. Lancet Oncol. 2016;17(5):671-680.

24. Li Y, Jia H, Yu W, et al. Nomograms for predicting prognostic value of inflammatory biomarkers in colorectal cancer patients after radical resection. Int J Cancer. 2016;139(1):220-231.

\section{Dovepress}

patient perspectives such as quality of life, adherence and satisfaction. The manuscript management system is completely online and includes a very quick and fair peer-review system, which is all easy to use. Visit http://www.dovepress.com/testimonials.php to read real quotes from published authors. 